\title{
Discovery of a Nonspecific Precancer Screening Indicator named "Dicentric+Translocation" of the Chromosomal Aberrations
}

\author{
Gang Liu* \\ Gansu Provincial Center for Disease Control and Prevention, China
}

*Corresponding author: Gang Liu, Radiology, Biomarker Laboratory, Joint Laboratory of Institute of Radiology, Chinese Academy of Medical Sciences, Lanzhou, Gansu, China.

To Cite This Article: Gang Liu, Discovery of a Nonspecific Precancer Screening Indicator named "Dicentric+Translocation" of the Chromosomal Aberrations. 2020 - 11(1). AJBSR.MS.ID.001610. DOI: 10.34297/AJBSR.2020.11.001610.

Received: 非 November 26, 2020; Published: 眥 December 09, 2020

\begin{abstract}
Background: To search the major carcinogenic factor or explore a non - specific early- stage and rapid screening indicators of cancer.

Methods: Select 49 tumor patients and 60 healthy persons, Chromosome aberration and lymphocyte micronucleus were analyzed before radiation therapy of tumor patients.

Results: the "Dicentric + Translocation" of Chromosome 36(73.47\%).The frequency of chromosome aberration and lymphocyte micronucleus were higher, especially the "Dicentric+Translocation "of chromosome aberrations $(\mathrm{P}<0.01)$, the frequency of double centromere chromosomes and translocation increased; the chromosome conglutination and dissolution appeared.

Conclusion: Could it be used as an early screening indicator for cancer; does it suggest that ionizing radiation is a major carcinogen? It is worthy of further study.
\end{abstract}

Keywords: Cancer screening indicators; Chromosome aberration analysis; Dicentric+Translocation chromosome analysis

\section{Background}

Identifying the causes of human cancer is the first step in cancer prevention. The identification of a cancer hazard may have broad and profound implications. As a disease with a truly global reach, cancer has touched most people in a personal way. Importantly, this new appreciation of cancer's complexity is redirecting attention to cancer prevention and early detection - research areas.

Cancer is not one disease, but many diseases. Cancer is a disease in which normal cells divide out of control and can invade other tissues. Cancer cells can spread to other parts of the body through the blood and lymphatic system. Cancer can be caused by many factors, such as exposure to carcinogens, genetic mutations, certain behaviors, age, and so on.

There are many ways to screen for cancer. Main types: physical examination and history: Examining the body for general signs of health, including signs of illness, such as a lump or other unusual object. The patient's living habits, past illnesses and treatment measures. Laboratory testing: Testing of human blood, tissue, urine or other substances. Imaging screening: Imaging of the internal areas of the body. Genetic test: Laboratory test that analyzes cells or tissues to look for genetic or chromosomal changes. These screening methods are used to find out whether a person is at risk for a particular disease or condition [1].

Cancer screening is looking for cancer before a patient has any symptoms. Screening is not meant to diagnose cancer. Screening can help detect cancer early, before symptoms appear. When abnormal tissue or cancer is caught early, it may be easier to treat or cure. Catching some cancers early (before symptoms appear) may help reduce the chance of dying from them. By the time symptoms appear, the cancer may have spread. This may make cancer more difficult to treat or cure.

In this study, chromosomal aberration analysis and lymphocyte micronucleus detection techniques were used to analyze the 
chromosomal aberration and lymphocyte micronucleus of cancer patients and normal people, in an attempt to explore a new cancer screening technology.

\section{Methods}

Reagents and Instruments: Biochemical RPMI-1640 (Xi'an, China), fetal bovine serum (FBS), and phytohemagglutinin-M were purchased from Invitrogen (GIBCO, Beijing), and potassium chloride and PI were obtained from Sigma Chemicals (Shanghai). Methanol and glacial acetic acid were obtained from Spectrochem (China). Quick CRP analyzer (Shanghai, China); Giemsa stain was purchased from Sigma-Aldrich (St Louis, Missouri), the SANYO MCO-20AIC CO2 incubator from SANY (Sakata, Japan), and microscope from OLYMPUS (OLYMPUS CK20, Tokyo, Japan).

\section{Blood samples}

a) Heparinized blood was collected from 49 patients with tumor (including stomach, colon, lung, cervical, and lymphomas cancers) and 60 healthy people.

b) The median age of patients in this trial was 62.95 years (range: 51-72 years; male: 42, female: 7).

c) The median age of healthy people in this trial was 58.21 years (range: $36-65$ years; male: 46 , female: 14 ).

d) This study was cleared by the Gansu Provincial Center for Disease Control and Prevention Ethical Committee and with the informed consent of patients.

\section{Cell culture and sample preparation}

Whole blood culture: Peripheral blood $2 \mathrm{ml}$ was collected from each cancer patients before radiation therapy and healthy people were divided into $0.5 \mathrm{~mL}$ portions and were added into $4 \mathrm{~mL}$ the culture media (80\% RPMI-1640 and 20\% FBS). The lymphocytes were cultured in Qingdao Laifo Biological Products Co. LTD 1640 culture medium containing FBS, 1\% penicillin sodium and $100 \mathrm{mg} /$ $\mathrm{mL}$ streptomycin, $0.45 \mathrm{~mL}$ heparin lithium was added into $4.5 \mathrm{~mL}$ lymphocyte culture medium as anticoagulant, and the proportion of blood and culture medium was $1: 10$, phytoagglutinin lutinin-M $(20 \mathrm{mg} / \mathrm{mL})$, and $0.04 \mathrm{~g} / \mathrm{mL}$ colchicines at $37^{\circ} \mathrm{C}$ in $5 \% \mathrm{CO} 2$ in a humidified incubator for 52 hours.

Table1: Chromosome aberration analysis.
Cell suspension was prepared: Cells were subjected to hypotonic treatment by $5 \mathrm{~mL} \mathrm{KCl}(0.075 \mathrm{M})$ solution one time 20 minutes, add $1 \mathrm{ml}$ of fixed solution (methanol/acetic acid 3:1) and fixed in advance once for 20 minutes, then fixed 3 times with fixed solution (methanol: glacial acetic acid 1:3) for 10 minutes each. Slides were produced. It was stained by giemsa staining, air-dried and coded. It was used to analyze the lymphocyte chromosome aberration $[2,3]$.

Peripheral blood $2 \mathrm{ml}$ was collected from each cancer patient and divided into $0.5 \mathrm{~mL}$ portions before radiation therapy and healthy people were distinguished; $0.5 \mathrm{~mL}$ of the blood was added to $4 \mathrm{~mL}$ portion of the culture media (80\% RPMI-1640 and $20 \%$ FBS). The mixture was stimulated with phytohemagg lutinin-M $(20 \mathrm{mg} / \mathrm{mL})$ and incubated at $37^{\circ} \mathrm{C}$ for 72 hours, the cells were harvested with a prechilled hypotonic solution $(0.075 \mathrm{M})$ and fixed with a carboy solution (methanol/acetic acid 3:1). Multiple slides were casted, air-dried, and coded. The slides were stained with $8 \%$ Giemsa solution in phosphate buffer ( $\mathrm{pH}$ 6.8, Propidium Iodine: 1 $\mathrm{mg} / \mathrm{mL}$ ). In the Giemsa-stained slides, we calculated the frequency of the micronuclei in the binucleated cells with intact cytoplasm using the criteria described in detail by Fenech. Lymphocyte blood counts were measured using Quick CRP analyzer (Hunan, China) [4].

\section{Statistical Methods}

The statistical analysis software SPSS version 10.0 was used for the analysis of variance and $t$ test for each corresponding groups.

“Dicentric+Translocation” Chromosome Analysis

All slides were used to analyze the lymphocyte chromosome aberration and micronucleus assay by the Olympus microscope; about 4360 metaphases were analyzed before RT and 6700 metaphases of healthy people were analyzed.

\section{Results}

Analysis of chromosome aberrations in peripheral blood lymphocytes the frequency of double centromere chromosomes and translocation increased; the conglutination and dissolution of chromosome appeared. The detection frequency of lymphocyte micronucleus increased (Table 1 \& 2).

\begin{tabular}{|c|c|c|c|c|c|c|c|}
\hline \multirow{2}{*}{ Subject } & \multirow{2}{*}{ Number } & \multirow{2}{*}{ Metaphases Scored } & \multicolumn{5}{|c|}{ Distribution } \\
\hline & & & $\mathbf{r}$ & dic & $\mathbf{t}$ & $\min$ & ace \\
\hline Before RT & 49 & 4360 & 0 & 30 & 6 & 4 & 2 \\
\hline Healthy people & 60 & 6700 & 0 & 0 & 0 & 0 & 12 \\
\hline$P$ & - & - & - & $<0.001$ & $<0.001$ & - & - \\
\hline
\end{tabular}

Abbreviation: dic: dicentric; r: centric rings; min: minute; t: translocation; ace: excess acentrics

The Analysis of chromosome aberrations in peripheral blood lymphocytes
Diacentric + translocation" (chromosome $36,73.47 \%$ ) was detected in 49 cases of tumor patients, and no "diacentric + 
translocation" chromosome was detected in healthy people. "Dicentric+Translocation" is a specific chromosomal aberration

caused by ionizing radiation, the background value is around $0.06 \%$.

\section{Detection and analysis of micronucleus in peripheral blood lymphocytes (Table 2)}

Table2: Lymphocyte micronucleus detection analysis.

\begin{tabular}{|c|c|c|c|c|c|}
\hline \multirow[t]{2}{*}{ Subject } & \multirow[t]{2}{*}{ Number } & \multicolumn{2}{|c|}{ Check out the Microkernel } & \multicolumn{2}{|c|}{ Micronucleus Cells } \\
\hline & & Number & $\%$ & Number & $\%$ \\
\hline Before RT & 98000 & $38 / 49$ & 77.55 & 85 & 0.97 \\
\hline Healthy people & 120000 & $25 / 60$ & 41.67 & 32 & 0.27 \\
\hline$P$ & & & $<0.001$ & & $<0.01$ \\
\hline
\end{tabular}

\section{Discussion}

Dicentric or translocation was detected in $73.47 \%$ of the cancer patient, the formation of dicentric or translocation is equivalent after DNA strand is broken. Dicentric or translocation was not detected in normal people.

Why are the "Dicentric+Translocation "frequently detected in cancer patients?

The Dicentric and Translocation are equivalent in chromosomal aberrations caused by ionizing radiation, because the chance of forming a dicentric or translocation is the same when the DNA double strand is broken by a ray.

Could it be used as an early screening indicator for cancer; does it suggest that ionizing radiation is a major carcinogen? It is worthy of further study. When there is limited evidence, a separate sentence identifies the target organ(s) or tissue(s) for which a positive association between exposure to the agent and the cancer(s) was observed in humans.

Evidence suggests that lack of carcinogenicity: There are several high-quality studies covering the full range of levels of exposure that humans are known to encounter, which are mutually consistent in not showing a positive association between exposure to the agent and the studied cancers at any observed level of exposure. The results from these studies alone or combined should have narrow confidence intervals with an upper limit below or close to the null value (e.g. a relative risk of unity). Bias and confounding were ruled out with reasonable confidence, and the studies were considered informative. A conclusion of evidence suggests that lack of carcinogenicity is limited to the cancer sites, populations and life stages, conditions and levels of exposure, and length of observation covered by the available studies. In addition, the possibility of a very small risk at the levels of exposure studied can never be excluded [5-8].

Although research on cancer screening and early detection is challenging, the potential payback makes it an important and valuable area of research in terms of preventing cancer deaths. With the development of many cancer potential biology, imaging technology, genetics and other areas of technological progress, for cancer screening and early detection of the development of new ways.

People who detect and treat cancer early improve survival or prolong survival compared with those who don't find the disease until symptoms appear. For many cancers, on the other hand, there are no good screening tests for early detection. There have been effective early screening tests, many of which have not been shown to be effective in reducing cancer mortality [9-14].

Many studies have found that screening has drawbacks as well as benefits. There is a risk of overdiagnosis and overtreatment-that is, of diagnosing and treating cancers that are not life threatening or causing symptoms. However, overdiagnosis and overtreatment unnecessarily expose patients to the potential physical harm of unnecessary and often invasive diagnostic tests and treatments, as well as the psychological stress associated with cancer diagnosis.

The current important work is how to improve the accuracy of cancer screening tests and expand the accuracy of cancer screening methods. There have been some success stories in screening and early detection. In the United States, for example, the death rate from cervical cancer has dropped significantly after annual pap smears, and colorectal and breast cancer screening has also been shown to reduce the death rate from these cancers.

Screening for cervical cancer and colorectal (colon) cancer can prevent cancer by finding early lesions so they can be treated or removed before they become cancerous. Screening for cervical, colorectal, breast, and lung cancers helps find these diseases at an early stage, when treatment works best.

Prevention is an important component in reducing cancer health disparities, including the higher incidence and mortality rates in some population groups Women undergoing radiation therapy for cervical or uterine cancer have a much higher incidence 
of serious side effects than previously realized, according to results from a new clinical trial. This information may help women and their clinicians more accurately weigh the potential benefits versus harms of adjuvant therapy, said the trial's leaders [16-18].

People can reduce their risk of getting cancer by making healthy choices like keeping a healthy weight, avoiding tobacco, limiting the amount of alcohol they drink, and protecting themselves.

For example, tobacco use is the leading preventable cause of cancer, contributing to at least 12 types of cancer, including acute myeloid leukemia (AML) and cancers of the oral cavity and pharynx; esophagus; stomach; colon and rectum; liver; pancreas; larynx; lung, bronchus, and trachea; kidney and renal pelvis; urinary bladder; and cervix [19-27].

\section{Conclusion}

The Dicentric and Translocation are equivalent in chromosomal aberrations caused by ionizing radiation, because the chance of forming a dicentric or translocation is the same when the DNA double strand is broken by a ray.

Could it be used as an early screening indicator for cancer; does it suggest that ionizing radiation is a major carcinogen? It is worthy of further study.

\section{Declarations}

Ethics approval and consent to participate.

The research was approved by the Bioethics Committee at the Gansu provincial center for disease control and prevention. Informed consent was obtained from all individual participants included in the study.

\section{Consent to publish}

No applicable.

\section{Availability of Data and Materials}

Data and materials are available.

\section{Competing Interests}

The authors declare that they have no conflict of interest.

\section{Funding}

This study was funded by the Lanzhou science and technology bureau (2018-1-127) and Gansu provincial health commission (GSWSKY2018-48).

\section{References}

1. Chen YZ, Fan ZH, Zhao YX, Lu B, Bao-Shen Z, et al. (2014) Singlenucleotide polymorphisms of LIG1 associated with risk of lung cancer. Tumor Biology 35(9): 9229-9232.

2. IAEA (2001) Cytogenetic analysis for radiation dose assessment Technical Report Series No. 405. Vienna, Austria.
3. Ministry of Health of the people's Republic of China (2011) GB/T 282362011 Method of Chromosome Aberration Analysis for Biological Dose Assessment(s). Standards Press of China, Beijing, China.

4. Fenech M (2007) Cytokinesis-block micronucleus cytome assay. Nature Protocols 2(5): 1084-1104.

5. Shayne MG, Jane SH, Brooke SC, Behnoosh M, Cheryll CT, et al. (2018) Surveillance for Cancers Associated with Tobacco Use - United States, 2010-2014. Surveillance Summaries 67(12): 1-42.

6. Wang S, The People's Hospital of Juye County (2014) Application of health education in the treatment of patients with cervical cancer. Journal of Qilu Nursing.

7. Yu-mei Z, Xian-zhi X; Shu-chun B, Ge-ping Y, Department of Gynecology and Obstetrics, et al. (2014) Epidemiological investigation and analysis of cervical cancer in 18365 elderly women in rural area of Dongying. Chinese Journal of Cancer Prevention and Treatment.

8. Wen-Hua Z, Xin-Hui Z, Li-Li G, Department of Preventive Medicine, Medical College of Shihezhi University, et al. (2014) Analysis of the results of rural women in cervical cancer screening program during 2009 to 2011 years in Xinjiang. Maternal and Child Health Care of China.

9. Alexandrov LB, Ju YS, Haase K, Van Loo P, Martincorena I, et al. (2016) Mutational signatures associated with tobacco smoking in human cancer. Science 354(6312): 618-622.

10. Axelson O, Steenland K (1988) Indirect methods of assessing the effects of tobacco use in occupational studies. Am J Ind Med 13(1): 105-118.

11. Baan RA, Stewart BW, Straif K (2019) Tumour site concordance and mechanisms of carcinogenesis. International Agency for Research on Cancer, Lyon, France.

12. Bieler GS, Williams RL (1993) Ratio estimates, the delta method, and quantal response tests for increased carcinogenicity. Biometrics 49(3): 793-801.

13. Capen CC, Dybing E, Rice JM, Wilbourn JD (1999) Species differences in thyroid, kidney and urinary bladder carcinogenesis. IARC Press, Lyon, France.

14. Guyton KZ, Kyle AD, Aubrecht J, Cogliano VJ, Eastmond DA, et al. (2009) Improving prediction of chemical carcinogenicity by considering multiple mechanisms and applying toxicogenomic approaches. Mutat Res 681(2-3): 230-240.

15. Guyton KZ, Rusyn I, Chiu WA, Corpet DE, van den Berg M, et al. (2018) Application of the key characteristics of carcinogens in cancer hazard identification. Carcinogenesis 39(4): 614-622.

16. Hanahan D, Weinberg RA (2000) The hallmarks of cancer. Cell 100(1): 57-70.

17. Hanahan D, Weinberg RA (2011) Hallmarks of cancer: the next generation. Cell 144(5): 646-674.

18. Haseman JK, Huff J, Boorman GA (1984) Use of historical control data in carcinogenicity studies in rodents. Toxicol Pathol 12(2): 126-135.

19. Herceg Z, Lambert MP, van Veldhoven K, Demetriou C, Vineis P, et al. (2013) Towards incorporating epigenetic mechanisms into carcinogen identification and evaluation. Carcinogenesis 34(9): 1955-1967.

20. Hernán MA, Hernández-Díaz S, Robins JM (2004) A structural approach to selection bias. Epidemiology 15(5): 615-625.

21. National Academies of Sciences, Engineering, and Medicine (2017) Using $21^{\text {st }}$ century science to improve risk-related evaluations. National Academies Press, Washington (DC), USA.

22. National Research Council (2012) Exposure science in the $21^{\text {st }}$ century: a vision and a strategy. National Academies Press, Washington (DC), USA. 
23. OECD (1997) Test no. 471: Bacterial reverse mutation test, OECD Guidelines for the Testing of Chemicals, Section 4. Organisation for Economic Co-operation and Development, Paris, France.

24. OECD (2016a). Test no. 476: In vitro mammalian cell gene mutation tests using the Hprt and xprt genes, OECD Guidelines for the Testing of Chemicals, Section 4. Organisation for Economic Co-operation and Development, Paris, France.

25. OECD (2016b) Test no. 490: In vitro mammalian cell gene mutation tests using the thymidine kinase gene, OECD Guidelines for the Testing of Chemicals, Section 4. Organisation for Economic Co-operation and Development, Paris, France.
26. OECD (2018) Test no. 451: Carcinogenicity studies, OECD Guidelines for the Testing of Chemicals, Section 4: Health effects. Organisation for Economic Co-operation and Development, Paris, France.

27. Vandenbroucke JP, Broadbent A, Pearce N (2016) Causality and causal inference in epidemiology: the need for a pluralistic approach. Int J Epidemiol 45(6): 1776-1786. 